\title{
Electromagnetic Structure Design Study of Fault-Tolerant Interior Permanent Magnet Machines for Electric Vehicles Using Harmonic Order Shaping
}

\author{
Guohai Liu, Yu Zeng, Wenxiang Zhao*, and Qian Chen \\ School of Electrical and Information Engineering, Jiangsu University, Zhenjiang 212013, China
}

(Received 3 September 2016, Received in final form 12 December 2016, Accepted 15 December 2016)

\begin{abstract}
Although pretty methods have been proposed to reduce torque ripple, they generally suffer from the decreased torque density. This paper will investigate the spoke-type interior permanent magnet (IPM) machine with shaping methods, including the sinusoidal (SIN), the inverse cosine (ICS), the sinusoidal with third harmonic (SIN+3rd), and the inverse cosine with third harmonic (ICS+3rd). In order to obtain low torque ripple and high torque density, the shaping method applied in rotor and stator at the same time, termed as the dualshaping method, is proposed. This method is analytically derived and further confirmed by finite element method (FEM). It turns out that the ICS and ICS+3rd shaping methods are more suitable for outer rotors, while the SIN and the SIN+3rd shaping method should be used in inner stators. The original machine, the singular shaped machines and the dual-shaped machines on electromagnetic performances are compared for evaluation. The results verify that the dual-shaping method can improve torque density, whilst reducing torque ripple.
\end{abstract}

Keywords : torque ripple, torque density, permanent magnet machine, shaping methods, finite element analysis

\section{Introduction}

At present, permanent magnet (PM) machines are shown increased interest due to electric vehicles (EVs) paid more attentions. Meanwhile, benefiting from high torque density, high efficiency and excellent dynamic performance, PM machines have been applied widely from rail transit to aerospace [1-3]. Interior PM (IPM) machines have gained more concerns for EVs because they have good fluxweakening capability and wide constant-power speed range $[4,5]$. Meanwhile, the fault-tolerant capacity is also considerably significant for EVs. In [6], an in-wheel faulttolerant interior permanent magnet (FT-IPM) machine with spoke-type topology was proposed for EVs. This machine offered high reliability owing to the multi-phase and fault-tolerant teeth [7-10]. In addition, fractional-slot and concentrated windings were often used in IPM machines in terms of low cogging torque and short end windings, thus, low copper loss and high efficiency [11].

However, concentrated fractional-slot windings as well as the thin air-gap length will bring about high harmonic

(C)The Korean Magnetics Society. All rights reserved.

*Corresponding author: Tel: +86-511-88722598

Fax: +86-511-88722596, e-mail: zwx@ujs.edu.cn contents in electromagnetic motive force (MMF). It causes many challenges for some high-performance applications requiring low torque ripple and high torque density. Hence, torque ripple reduction is necessary for IPM machines to achieve smooth operation. Torque ripples mainly arise from the cogging torque and non-ideal sinusoidal back electromotive force (EMF). The cogging torque is caused by the interaction between PMs and slots, while the back-EMF is influenced by the air-gap flux density. In addition, torque ripples are also affected by magnetic saturation in the stator and rotor under load condition. What's more, the reluctance torque caused by the difference between the $d$-axis inductance and the $q$-axis inductance also affects torque ripples. Then, it can be concluded that the back-EMF, cogging torque and reluctance torque have significant effect upon the torque ripple [12].

Different approaches have been proposed to reduce torque ripples, such as the ratio of pole arc to pole pitch [13], rotor outer surface shaping [14], and skew [15]. In addition, the sinusoidal distribution of air-gap flux density can be achieved by the rotor steel shaping. It is regrettable that these methods suffer from the decreased torque density. Then, the sinusoidal with third harmonic $(\mathrm{SIN}+3 \mathrm{rd})$ [16] and the inverse cosine with third harmonic (ICS+3rd) [17] shaping methods were proposed to improve the torque 
density. However, there are some limitations of two proposed shaping methods. Ignoring the minor things, the main problem was that they only can be applied in inner rotor, while outer rotor, inner stator and outer stator are not taken into consideration. In addition, only the singular shaping was studied, i.e., the shaping methods were only employed in stator or rotor alone, while both stator and rotor shapes were not investigated.

In this paper, the dual-shaping method is proposed to achieve low torque ripple and high torque density. Four shaping methods including the SIN, the ICS, the SIN+3rd, and the ICS $+3 \mathrm{rd}$, respectively applied in rotor and stator at the same time, are investigated. In Section 2, the topologies of the spoke-type IPM machine with various shaping methods will be described, whilst the operating principle and formula derivation will be also given. The electromagnetic performances of the original machine and the proposed machines will be compared and analyzed in Section 3. Finally, the conclusion will be drawn in Section 4.

\section{Spoke-Type IPM Machines with Different Shaping Methods}

\subsection{Configuration of Original Machine}

A 20-slot/18-pole fault-tolerant spoke-type machine is introduced to investigate the influence of shaping methods as shown in Fig. 1. It can be seen that the inner stator with concentrated fractional-slot windings is employed.

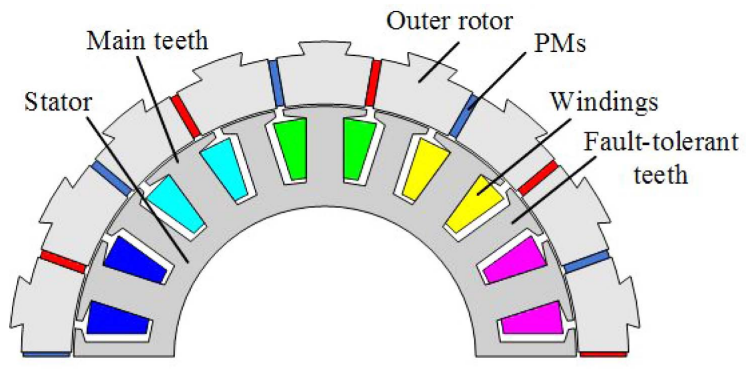

(a)

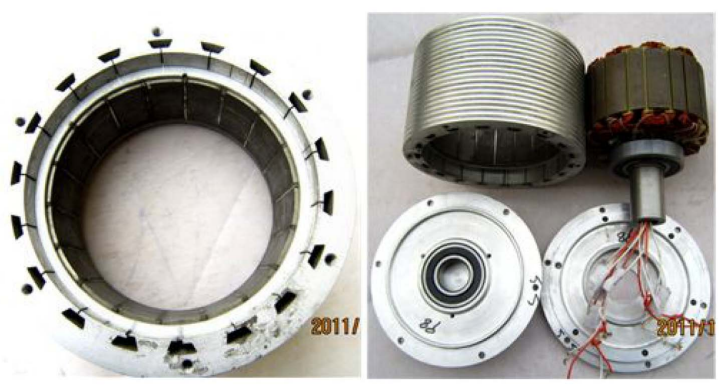

(b)

Fig. 1. (Color online) Original spoke-type machine. (a) Topology. (b) Prototype.

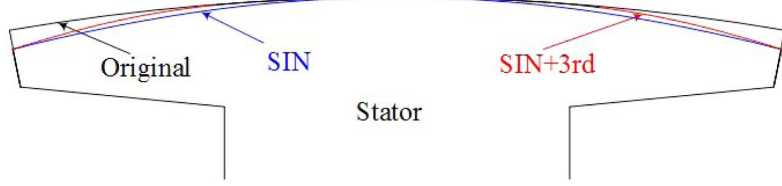

Fig. 2. (Color online) Shaped stators.

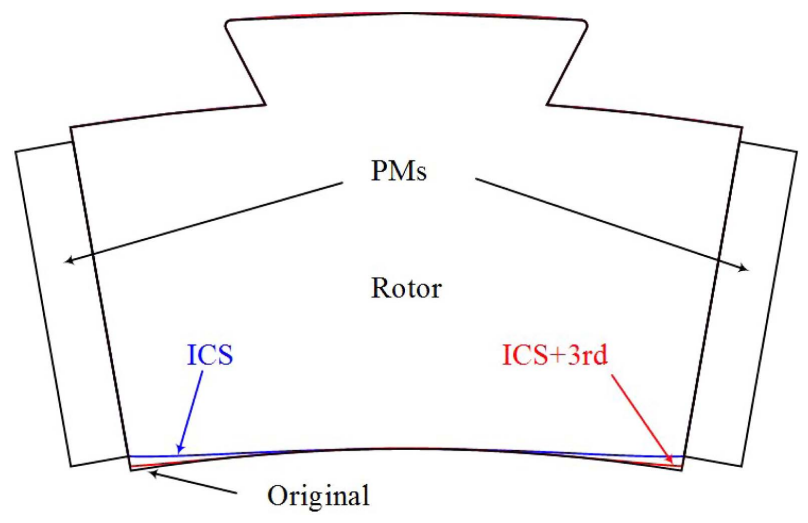

Fig. 3. (Color online) Shaped rotors.

Meanwhile, the five-phase and the fault-tolerant teeth (FFT) are used to improve the fault-tolerance and stability. The proposed stators with different contours are designed in Fig. 2. Three contour lines are respectively described by the original stator, the SIN stator and the SIN+3rd stator. In Fig. 3, spoke-type rotors with different profile shapes are presented. Three contour lines are described by the original rotor, the ICS rotor and the ICS +3 rd rotor, respectively. It is worth mentioning that the minimum airgap lengths of different shaped machines are the same, i.e., $0.5 \mathrm{~mm}$. It means the equivalent air-gap length of the shaped machines in one electrical period is increased compared with the original machine. This brings about the increased equivalent reluctance which leads to the torque density reduction. Hence, the torque ripple and torque density are restricted each other.

\subsection{Shaping Methods Employed in Rotor and Stator}

Shaping methods employed in stator and rotor are shown in Fig. 4. The steel thickness $h(\theta)$ varying with the position $\theta$ is described by the $\mathrm{SIN}+3 \mathrm{rd}$ shaping method as shown in Fig. 2, while the air-gap length $g(\theta)$ changing with the rotor position $\theta$ is achieved by the ICS +3 rd shaping method as seen in Fig. 3. The curve of $l_{1}$ represents the SIN shaping, whilst the $\mathrm{SIN}+3 \mathrm{rd}$ shaping is described by $l_{4}$ which is overlaid by $l_{2}$ and $l_{3}$. The curves of $l_{2}$ and $l_{3}$ are the fundamental harmonic and the third harmonic, respectively. If $l_{1}$ and $l_{4}$ have the same amplitude, the fundamental harmonic of $l_{4}$ will be higher than 


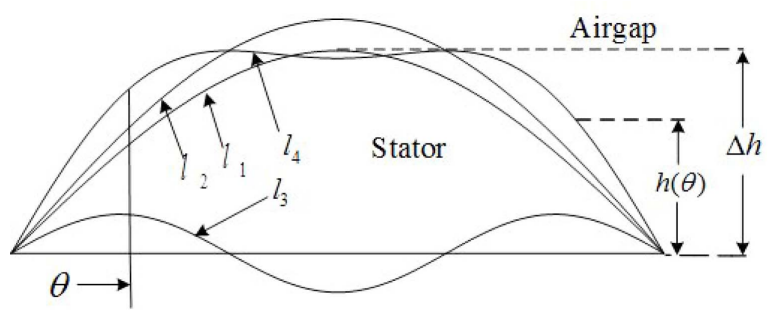

(a)

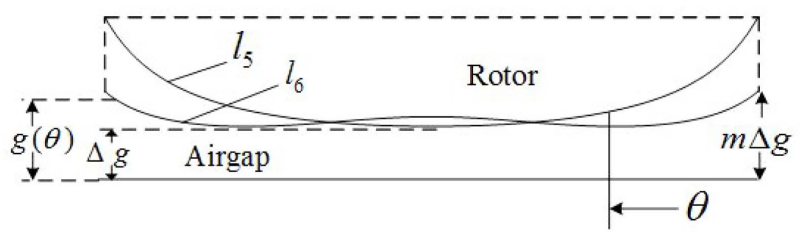

(b)

Fig. 4. Shaping methods. (a) SIN method and SIN +3 rd method. (b) ICS method and ICS +3 rd method.

$l_{1}$, i.e., the amplitude of $l_{2}$ is larger than $l_{1}$. The higher fundamental harmonic certainly results in torque density improvement. In addition, the equivalent air-gap length of $l_{4}$ is smaller than $l_{1}$ under the same amplitudes. Then, the equivalent reluctance of $l_{4}$ is lower than $l_{1}$ too. Hence, the torque density will be improved further from $l_{1}$ to $l_{4}$. Foregoing analyses show essential mechanisms of torque density improvement from the SIN method to the SIN+3rd method. In Fig. 4(b), the contours of $l_{5}$ and $l_{6}$ represent the ICS and the ICS +3 rd shaping methods, respectively. The illustrations of Fig. 4(b) are simplified because the related analyses are similar with those of Fig. 4(a). $l_{5}$ only has the fundamental harmonic, while $l_{6}$ contains the fundamental harmonic and the third harmonic which are not marked in the figure though. If $l_{5}$ and $l_{6}$ have the same amplitudes, the fundamental harmonic of $l_{6}$ will be higher than that of $l_{5}$. Meanwhile, $l_{6}$ has the lower air-gap length compared with $l_{5}$. Therefore, the torque density of the ICS +3 rd method will be increased compared with that of the ICS method. It is worth mentioning that $\Delta g$ is a constant, i.e., $0.5 \mathrm{~mm}$. This result from the minimum airgap lengths of different shaped machines are the same as the original machine.

In order to gain the sinusoidal air-gap flux density, rotor and stator need to follow a specific function. The variations of the SIN and the ICS have been derived and given as (1) and (2), respectively

$$
\begin{aligned}
& h(\theta)=\Delta h \sin (p \theta) \\
& g(\theta)=\frac{\Delta g}{\cos \left(\frac{\pi}{\tau_{p}} \theta\right)}
\end{aligned}
$$

where both of them are functions of space position $\theta$. $\Delta h$ is the maximum thickness of PM or steel, while $\Delta g$ is the minimum air-gap length. $p$ and $\tau_{p}$ are the pole-pair number and the pole pitch of PM rotors, respectively.

However, the SIN and the ICS shaping methods suffer from the decreased torque density in spite of the torque ripple being reduced. Aiming at improving torque density, the SIN+3rd [16] and the ICS+3rd [17] have been proposed. The equations are given by (3) and (4), respectively

$$
\begin{aligned}
& h(\theta)=k_{h} \Delta h[\sin (p \theta)+a \sin (3 p \theta)] \\
& g(\theta)=\frac{k_{g} \Delta g}{\cos \left(\frac{\pi}{\tau_{p}} \theta\right)-a \cos \left(3 \frac{\pi}{\tau_{p}} \theta\right)}
\end{aligned}
$$

where $k_{h}$ and $k_{g}$ are the parameters to be determined. $a$ is the amplitude of injected third harmonic. $k_{h}$ varies with the value of the injected third harmonic in order to maintain the same maximum PM or steel thickness, while $k_{g}$ changes with the injected third harmonic to obtain the same minimum air-gap length.

However, there are three weaknesses of four mentioned shaping methods. At first, these methods are only employed in inner rotor, while outer rotor, inner stator and outer stator are not taken into consideration. The next, these shaping methods are applied to stator or rotor alone, while both stator and rotor shapes are not investigated. Finally, four shaping methods only can be used in PM machines due to the typical parameters of $p$ and $\tau_{p}$. It means the shaping methods are not accepted by the nonPM machines. In order to overcome these shortages, the new formulas are proposed as shown in (5) and (6)

$$
\begin{aligned}
& h(\theta)=k_{h} \Delta h[\sin (b \theta)+a \sin (3 b \theta)] \\
& g(\theta)=\frac{k_{g} \Delta g}{\cos (b \theta)-a \cos (3 b \theta)}
\end{aligned}
$$

where parameter $b$ is proposed to adjust the shaped arcs of stator and rotor in one electric period, whilst its value is given in following derivation. It is worth mentioning that when values of $k_{h}, k_{g}$ and $a$ are respectively 1,1 and 0 , the SIN and the ICS shaping methods are represented by equations (5) and (6), respectively, because the third harmonic is not injected.

In order to illustrate new shaping methods clearly, the shaped stator of the spoke-type machine is introduced. The functions of (1)-(4) cannot be applied in this machine because the necessary parameters $p$ and $\tau_{p}$ do not exist in inner stator as shown in Fig. 2. When the proposed formulas (5) and (6) are employed, the SIN and the ICS shaping methods are considered firstly because they are the basis of the third harmonic injection, i.e., $k_{h}=1, k_{g}=1$ 
and $a=0$. The arc of the stator tooth is easy to know as $\theta$. Then, the maximum steel thickness and air-gap length are respectively given by

$$
\begin{aligned}
& h(\theta)_{\max }=\left\{k_{h} \Delta h[\sin (b \theta)+a \sin (3 b \theta)]\right\}_{\max }=\Delta h \\
& g(\theta)_{\max }=\left[\frac{k_{g} \Delta g}{\cos (b \theta)-a \cos (3 b \theta)}\right]_{\max }=m \Delta g
\end{aligned}
$$

where $m$ is the multiples of maximum to minimum airgap lengths as shown in Fig. 4(b). Based on above conditions, the values of $b$ in equations (5) and (6) can be derived as

$$
\begin{aligned}
& b=\frac{\arcsin 1}{\theta} \\
& b=\frac{\arccos m}{\theta} .
\end{aligned}
$$

In addition, by solving the equation (7), the maximum PM thickness occurs at

$$
\cos (b \theta)=\left(\frac{9 a-1}{12 a}\right)^{\frac{1}{2}} \text {. }
$$

From (7) and (11), $k_{h}$ can be derived as

$$
k_{h}=\frac{1}{8 a}\left(\frac{1+3 a}{12 a}\right)^{-\frac{3}{2}}
$$

Generally, for the sinusoidal structure of PM or steel, the equivalent air-gap length will be increased compared with the original machine. This brings about the increased equivalent reluctance which leads to the torque density reduction. Hence, the third harmonic injection is employed to improve average torque, as shown in Fig. 4. Theoretically, the minimum equivalent area of air-gap, i.e., the maximum area of PM or steel, will possess the maximum average torque. For the SIN +3 rd shaping method, the value of $b$ is given by (9). Then, its area of PM or steel in half period can be derived as

$$
S_{h}=\int_{0}^{T / 2} h(\theta) d \theta=\int_{0}^{T / 2} k_{h} \Delta h[\sin (b \theta)+a \sin (3 b \theta)] d \theta .
$$

By applying the theory of Lagrange for optimization [16], to maximize the area of (11), while maintaining the same maximum thickness of (7). The function involved with the amplitude of third harmonic $a$ can be expressed as

$$
\begin{aligned}
L\left(k_{h}, a\right)= & \int_{0}^{T / 2} k_{h} \Delta h[\sin (b \theta)+a \sin (3 b \theta)] d \theta \\
& +\lambda\left(k_{h}-\frac{1}{8 a}\left(\frac{1+3 a}{12 a}\right)^{-\frac{3}{2}}\right)
\end{aligned}
$$

Table 1. Shaping parameters of the spoke-type machine.

\begin{tabular}{lc}
\hline \hline \multicolumn{1}{c}{ Parameters } & Values \\
\hline Teeth arc $\theta_{1}$ & $19.3^{\circ}$ \\
Fault-tolerant teeth arc $\theta_{2}$ & $12.9^{\circ}$ \\
Core arc of rotor $\theta_{3}$ & $18.1^{\circ}$ \\
The maximum shaping length $\Delta h$ & $0.5 \mathrm{~mm}$ \\
The minimum air-gap length $\Delta g$ & $0.5 \mathrm{~mm}$ \\
$k$ under the SIN stator or the ICS rotor & 1 \\
$k$ under the SIN+3rd stator & $2 \sqrt{3} / 3$ \\
$k$ under the ICS+3rd rotor & $\sqrt{3} / 2$ \\
$a$ under the SIN stator or the ICS rotor & 0 \\
$a$ under the SIN+3rd stator or the ICS +3 rd stator & $1 / 6$ \\
\hline
\end{tabular}

where $\lambda$ is the Lagrange factor. Hence, the maximum torque of SIN +3 rd shaping method can be identified by differentiating $L\left(k_{h}, a\right)$ with respect to $k_{h}$ and $a$. Solving the equation (12), the values of $a$ and $k_{h}$ are derived as

$$
\begin{aligned}
& a=\frac{1}{6} \\
& k_{h}=\frac{2 \sqrt{3}}{3} .
\end{aligned}
$$

Similarly, the ICS +3 rd shaping method obtain the same value of $a$, i.e., $1 / 6$. It has been verified in [17] by finite element method, whilst from (8), the value of $k_{g}$ is given by

$$
k_{g}=\frac{\sqrt{3}}{2} .
$$

Table 1 shows detailed shaping parameters of the spoke-type machine. Together with shaping formulas (5) and (6), the shaped contours of stator teeth can be described in Fig. 2, whilst Fig. 3 presents the shaped contours of iron rotor core. From Table 1, it should be noted that $\theta_{1}, \theta_{2}$ and $\theta_{3}$ are different for various machines. $\Delta h$ in the SIN shaping method can be optimized by FEM. $\Delta h$ in the SIN+3rd shaping formula will keep the same as the SIN shaping, whilst the ICS +3 rd and the ICS shaping formulas have the same $\Delta g$, i.e., $0.5 \mathrm{~mm}$. In addition, the remaining parameters are constant for different machines because they are analytically derived.

\section{Various Shapes and Driving Performance Comparison}

\subsection{SIN Rotor and ICS Rotor}

Owing to the structure of outer rotor, the SIN and the ICS shaping methods used in the spoke-type rotors have totally different impacts. From (3) and (4), $h(\theta)$ is increased along with the increased $\theta$, whilst its maximum value is 
$\Delta h . g(\theta)$ is also increased with the increased $\theta$, while its maximum value is infinite when $\theta$ reach to $\pi / 2 b$. It means the growth rate of the ICS formula is much higher than the SIN formula. Thus, the ICS and the ICS +3 rd are more suitable for outer rotor shapes because the outer rotor has lower curvature compared with the inner rotor.

In order to verify the theoretical analysis, FEM is employed to predict the electromagnetic performances of the shaped machines. The original rotor, the SIN rotor and the ICS rotor with the same stator are compared. The back-EMFs of three rotors are given in Fig. 5. It can be seen that the ICS rotor gains the most sinusoidal backEMF under the similar amplitudes, while the original rotor and the SIN rotor are relative terrible. Figure 6 shows the cogging torques of three rotors. The ICS rotor owes the minimum cogging torque, while the original rotor has the highest cogging torque. The most sinusoidal back-EMF and minimum cogging torque of the ICS rotor are certainly leading to the minimum torque ripple. It is verified in Fig. 7, which compares output torques of three rotors. The torque ripple of the ICS rotor is reduced by $7.2 \%$, from $12.5 \%$ to $5.3 \%$, whilst its average torque is similar with the original rotor. By contrast, although the torque ripple of the SIN rotor is reduced by $3.8 \%$ com-

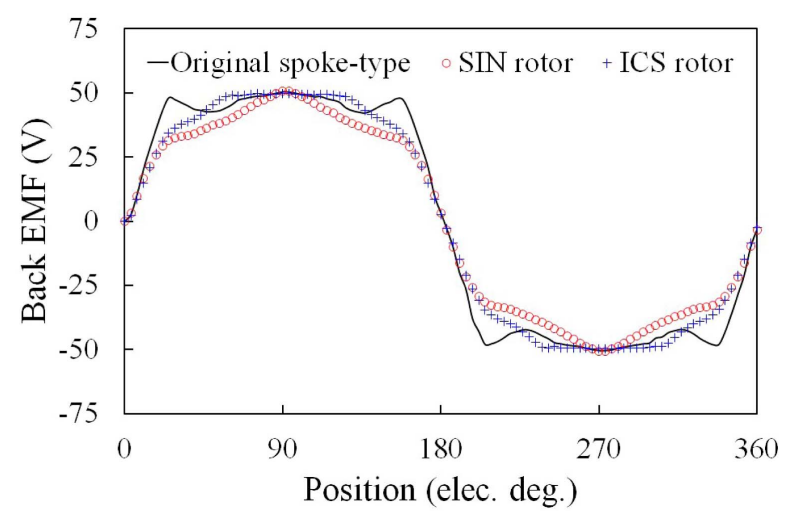

Fig. 5. (Color online) Back-EMFs of SIN and ICS rotors.

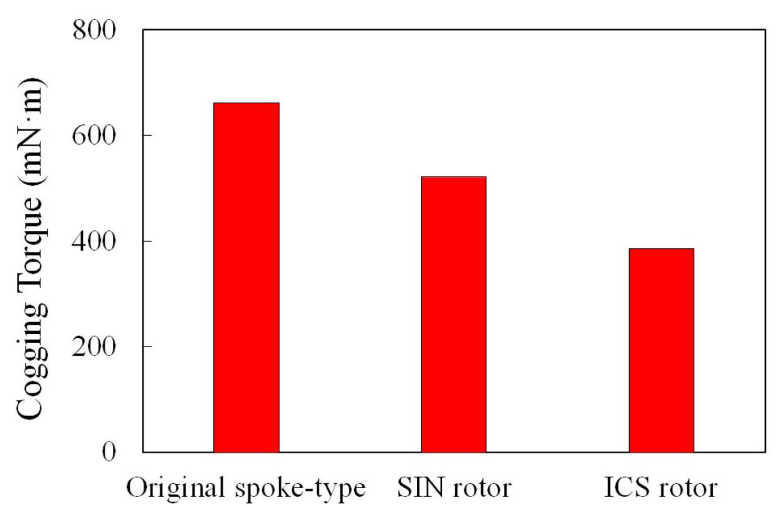

Fig. 6. (Color online) Cogging torques of SIN and ICS rotors.

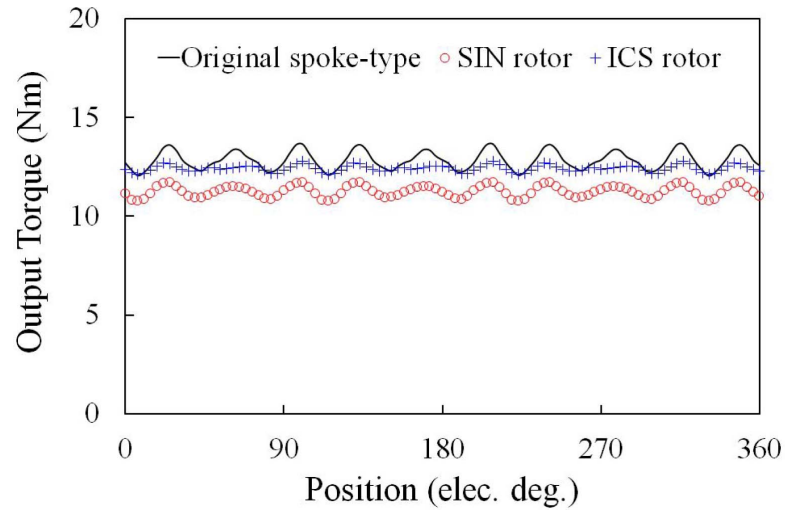

Fig. 7. (Color online) Output torques of SIN and ICS rotors.

pared with the original rotor, the average torque is reduced by $12.4 \%$. This is an unacceptable result due to the significant average torque reduction. Hence, it can be concluded that the ICS shaping method is more suitable for outer rotor compared with the SIN shaping method. In addition, for the ICS +3 rd shaping method, the same conclusion can be obtained, i.e., the ICS +3 rd shaping method is more suitable for outer rotor compared with the SIN+3rd shaping method because the third harmonic injection is based on the SIN and the ICS shaping methods. Therefore, the ICS and the ICS+3rd shaping methods are suitable to be employed in outer rotors, while the SIN and the $\mathrm{SIN}+3 \mathrm{rd}$ shaping methods are used in inner stators due to the higher curvature.

\subsection{Singular Shaping Methods}

Under the guidance of above analyses, four situations can be concluded when four shaping methods employed in the spoke-type machine, including the SIN stator, the $\mathrm{SIN}+3$ rd stator, the ICS rotor and the ICS +3 rd rotor. In order to obtain a clear conclusion, the analysis of electromagnetic performance is simplified. Output torques are

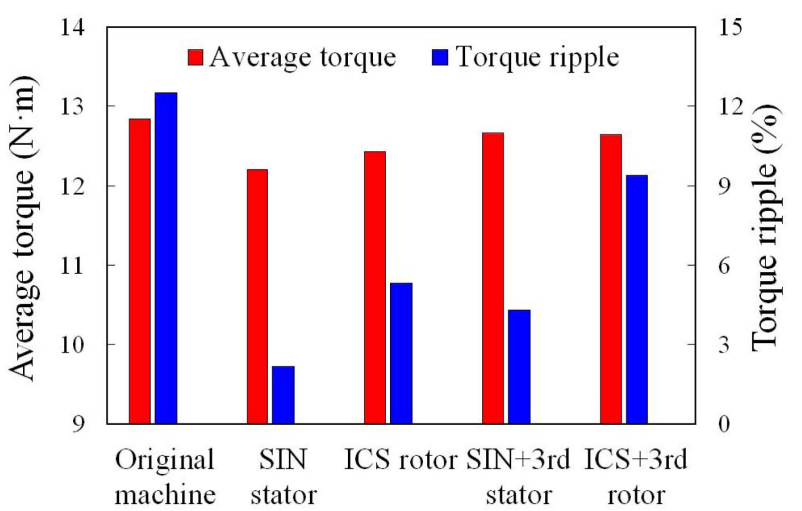

Fig. 8. (Color online) Average torques and torque ripples under singular shaping. 
considered as a main standard to assess merits and drawbacks of four shaped machines.

Figure 8 compares average torques and torque ripples of four shaped machines. It can be seen that the average torque and torque ripple of the original machine are the highest, while those of the SIN stator are the lowest. This verifies that the average torque is considerably compromised when the torque ripple reduction method is employed. In addition, the average torque and torque ripple of the ICS rotor are just increased a little compared with those of the SIN stator. Hence, the ICS rotor is not the candidate of the best output torque owner among four shaped machines. Focusing on the SIN+3rd stator, although its torque ripple is slightly increased, the average torque is enhanced significantly. Meanwhile, its average torque is the maximum one. For the ICS +3 rd rotor, the average torque is lower than the $\mathrm{SIN}+3$ rd stator and its torque ripple is also higher. Under the comprehensive consideration, it can be identified that the $\mathrm{SIN}+3 \mathrm{rd}$ stator possesses the best output torque. Therefore, the $\mathrm{SIN}+3 \mathrm{rd}$ stator will be selected to compare with the dual-shaped machines.

\subsection{Dual-Shaping Methods}

The dual-shaped machines will be compared for evaluation in this subsection. Four groups of comparing schemes can be proposed including the SIN stator and the ICS rotor (machine I), the SIN stator and the ICS +3 rd rotor (machine II), the SIN +3 rd stator together with the ICS rotor (machine III) and the SIN+3rd stator together with the ICS +3 rd rotor (machine IV). Output torques are still considered as the standard to assess the performance of the shaped machines. The SIN+3rd stator with the best output torque in singular shaped machines is added to illustrate the superiority of the dual-shaping method.

Average torques and torque ripples of five shaped

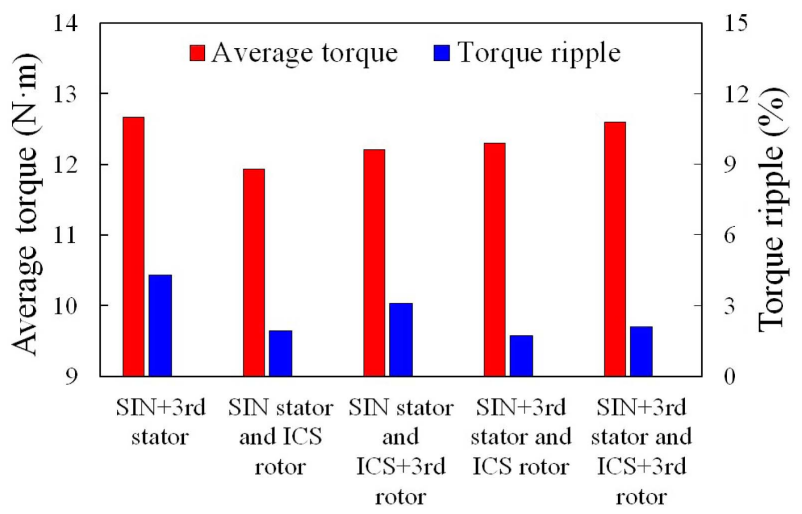

Fig. 9. (Color online) Average torques and torque ripples under dual-shaping. machines are shown in Fig. 9. Four dual-shaping machines all have low torque ripple especially for the machine III. Meanwhile, the torque densities of four dual-shaped machines are reduced a little compared with the singular shaped machine except machine IV. This is because both shaped stator and rotor lead to the increased average airgap length. Then, the equivalent reluctance is enhanced as well. Hence, the harmonic content of air-gap flux density is reduced especially for the fundamental one. It causes the decreased torque density. However, machine IV gains the most balanced performance which contains the lowest torque ripple and relatively high average torque. Hence, this machine will compare with the original machine in detail.

\subsection{Performance Comparison of Different Shapes}

In order to illustrate the merits of the dual-shaping method, four machines are compared, including the original machine, machine IV, the machine with the SIN stator and the machine with the SIN +3 rd stator. Figure 10 shows back-EMFs of four machines. It can be seen that machine IV gains the most sinusoidal curve among four

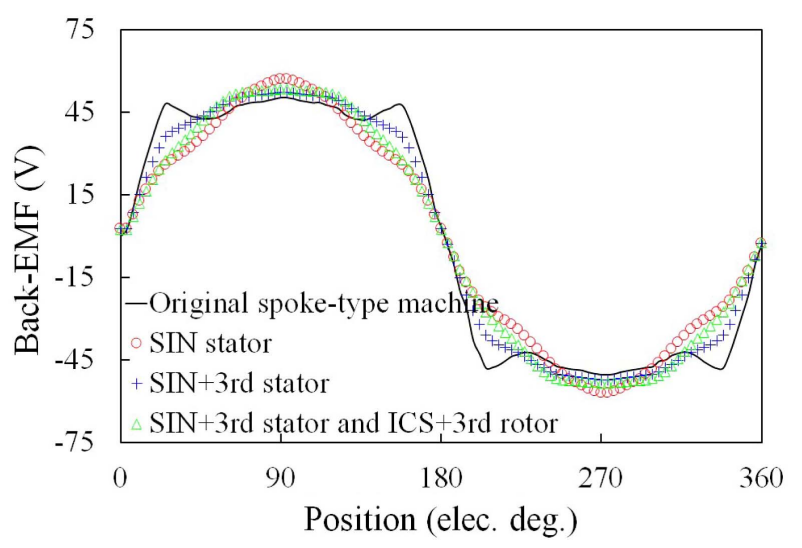

Fig. 10. (Color online) Comparison of back-EMFs.

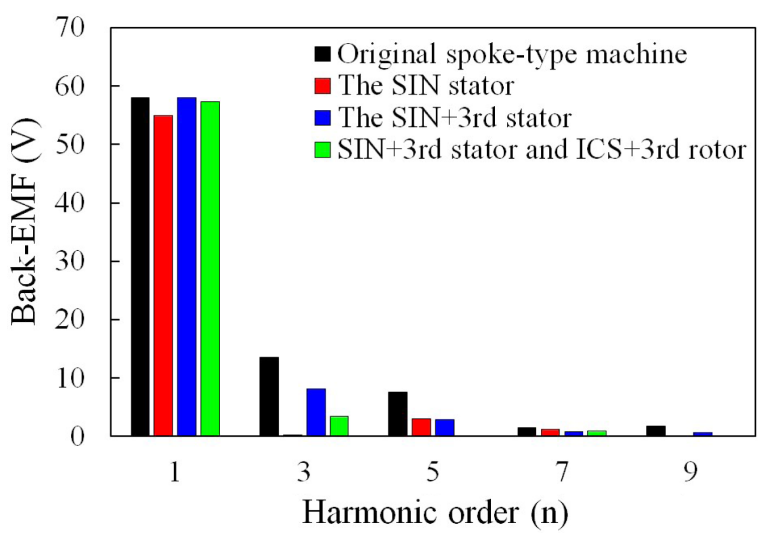

Fig. 11. (Color online) Harmonic analysis of back-EMFs. 
machines, while the SIN stator has the highest amplitude. However, it does not mean the SIN stator obtains the maximum value of the fundamental harmonic. It can be verified in Fig. 11 which shows the harmonic analysis of back-EMFs. The highest back-EMF of the SIN stator is caused by the fifth order harmonic, while its fundamental harmonic is lowest. The fundamental harmonic of machine IV and the SIN+3rd stator are both improved compared with that of the SIN stator, whilst their amplitudes are similar to the original machine. The third and fifth harmonics in the SIN+3rd stator are increased a lot, while the corresponding increments in machine IV are very low. Theoretically, only the fundamental harmonic contributes to average torque and the remaining harmonics will cause torque ripple. Hence, the lower total harmonic distortion of the back-EMF in machine IV is certainly leading to the smaller torque ripple.

Another vital cause of torque ripples is the cogging torque. Figure 12 compares the cogging torques of four machines. The cogging torques of three shaped machines are much lower than the original machine, whilst that of machine IV is the lowest. Machine IV, with the high

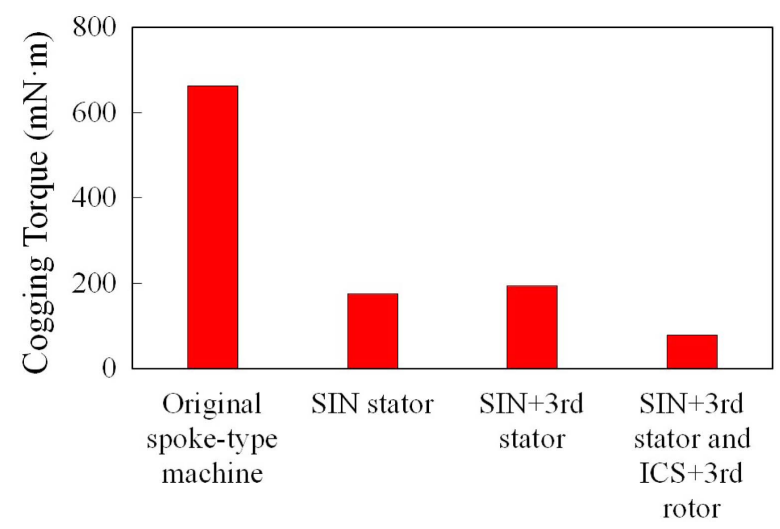

Fig. 12. (Color online) Comparison of cogging torques.

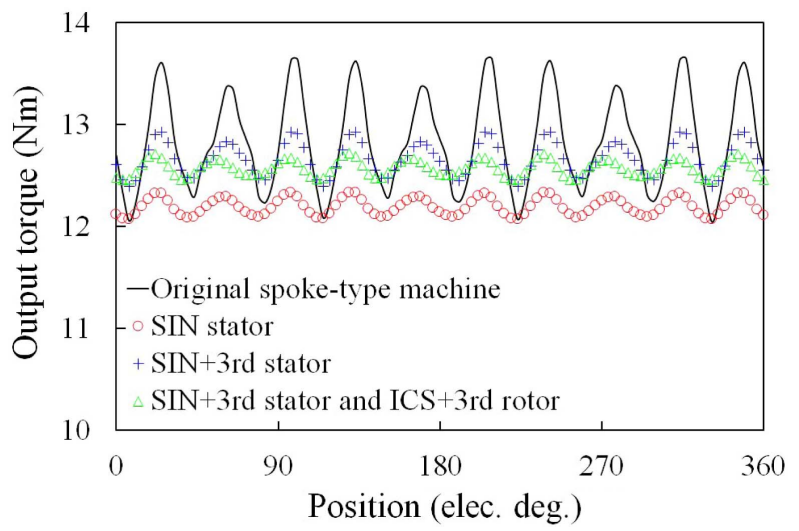

Fig. 13. (Color online) Comparison of output torques.

fundamental harmonic, low harmonic distortion and low cogging torque, obviously result in good output torque. Figure 13 shows the comparison of output torques. Compared with the original machine, although the torque ripple of the SIN stator is reduced by $10.3 \%$, from 12.5 $\%$ to $2.2 \%$, its average torque is decreased by $5 \%$ as well. Also, machine IV, the original machine and the $\mathrm{SIN}+3 \mathrm{rd}$ stator have similar average torques, but machine IV has the minimum torque ripple among three machines, only $2.1 \%$. Therefore, machine IV possesses high torque density and low torque ripple. This conclusion proves that the dual-shaping method is an effective way to improve torque performance.

Detailed comparisons of electromagnetic performances are given in Table 2. Benefiting from low back-EMF harmonics as shown in Fig. 11, the SIN stator and machine IV have lower core loss compared with the original machine. However, the eddy losses of four proposed machines are almost the same due to the spoke-type topology and the low volume of PMs. Under the operating condition with $1500 \mathrm{rpm}$ rated speed and 14.1 A peak current, these

Table 2. Comparisons of electromagnetic performances.

\begin{tabular}{|c|c|c|c|c|}
\hline & $\begin{array}{c}\text { Original spoke-type } \\
\text { machine }\end{array}$ & SIN stator & $\mathrm{SIN}+3$ rd stator & $\begin{array}{l}\text { SIN+3rd stator } \\
\text { and ICS }+3 \text { rd rotor }\end{array}$ \\
\hline Rated speed (rpm) & 1500 & 1500 & 1500 & 1500 \\
\hline Peak current (A) & 14.1 & 14.1 & 14.1 & 14.1 \\
\hline Fundamental harmonic of back-EMF (V) & 58.0 & 54.9 & 58.0 & 57.3 \\
\hline Cogging torque $(\mathrm{mN} \cdot \mathrm{m})$ & 622.4 & 175.0 & 193.0 & 78.0 \\
\hline Average torque $(\mathrm{N} \cdot \mathrm{m})$ & 12.8 & 12.2 & 12.6 & 12.6 \\
\hline Torque ripple (\%) & 12.5 & 2.2 & 4.3 & 2.1 \\
\hline Core loss (W) & 85.0 & 72.0 & 77.0 & 73.0 \\
\hline Eddy loss (W) & 1.6 & 1.5 & 1.5 & 1.5 \\
\hline Copper loss (W) & 45.6 & 45.6 & 45.6 & 45.6 \\
\hline Efficiency (\%) & 93.4 & 93.8 & 93.7 & 94.0 \\
\hline
\end{tabular}




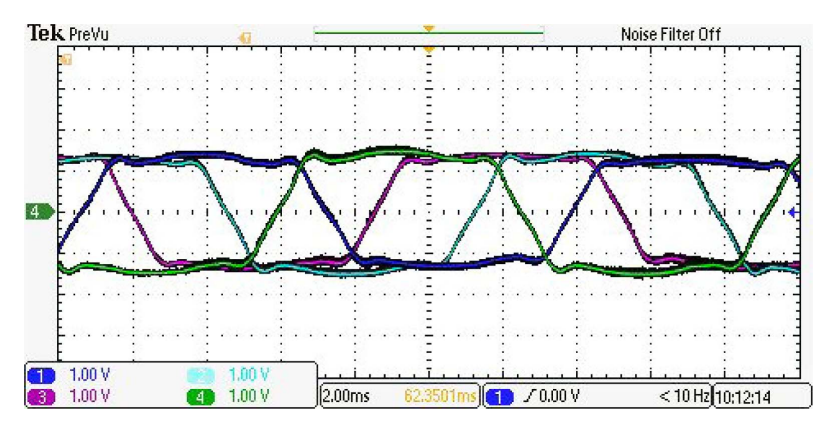

Fig. 14. (Color online) Measured phase back-EMFs.

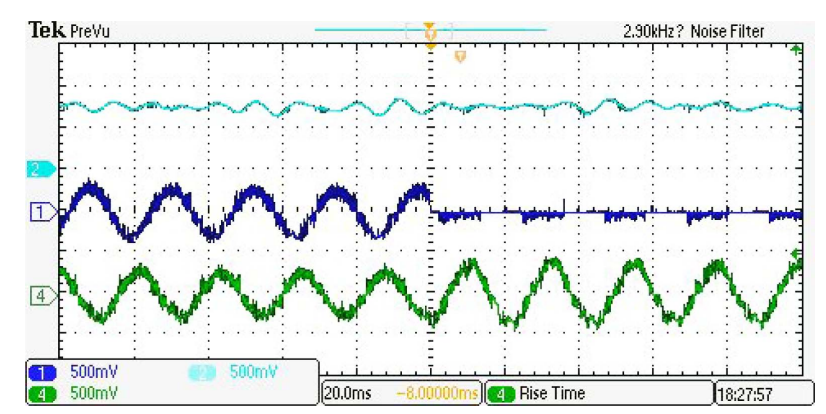

Fig. 15. (Color online) Torque, current of open circuit, and current of health $(1 \mathrm{~N} \cdot \mathrm{m} / \mathrm{div}, 5 \mathrm{~A} / \mathrm{div})$.

good electromagnetic performances, including the high average torque, low core loss, low eddy loss and the same copper loss of machine IV, cause the highest efficiency, i.e., $94.0 \%$.

\subsection{Experiment and Discussion}

The 18-pole/20-slot spoke-type experimental machine (original machine) is manufactured and measured to verify the theoretical analysis. The prototype is given in Fig. 1. It can be observed that the FFT are adopted to improve the fault-tolerance and stability.

Figure 14 shows the experimental results for the phase back-EMF of the original machine, which agree with the predicted results in Figs. 5 and 10. The torque $\left(T_{e}\right)$, current of open circuit $\left(i_{a}\right)$, and current of health $\left(i_{e}\right)$ under the remedial control strategy are shown in Fig. 15. It can be seen that the measured output torque (average torque and torque ripple) are almost the same under the normal and open-circuit fault. The fault tolerance of the original machine can be verified.

\section{Conclusion}

In this paper, four shaping methods employed in the fault-tolerant spoke-type IPM machine are investigated including the SIN, the SIN +3 rd, the ICS and the ICS +3 rd. The topologies of spoke-type machines with various shap- ing contours are introduced. Meanwhile, new formulas are derived to achieve wider applications. Namely, the SIN rotor and the ICS rotor are studied. It turns out that the ICS and ICS+3rd shaping methods are more suitable to outer rotors, while the SIN and the SIN+3rd shaping method should be used in inner stators. The next, the singular shaped and dual-shaped machines are compared. Their merits and drawbacks are presented in detail. Finally, the original machine, the optimal dual-shaped machine and two singular shaped machines are further compared for evaluation. The results prove that the dualshaping method is the effective method to achieve low torque ripple and high torque density.

\section{Acknowledgement}

This work was supported by the National Natural Science Foundation of China (51422702 and 51577084), by the Research Fund for 333 Project of Jiangsu Province (Project BRA2015302), by the Key Project of Natural Science Foundation of Jiangsu Higher Education Institutions (15KJA470002), and by the Priority Academic Program Development of Jiangsu Higher Education Institutions.

\section{References}

[1] M. Z. Ahmad, E. Sulaiman, and T. Kosaka, J. Magn. 20, 265 (2015).

[2] D. W. Chung and Y. M. You, J. Magn. 20, 176 (2015).

[3] W. P. Cao, B. C. Mecrow, G. J. Atkinson, J. W. Bennett, and D. J. Atkinson, IEEE Trans. Ind. Electron. 59, 3523 (2012).

[4] F. Parasiliti, M. Villani, S. Lucidi, and F. Rinaldi, IEEE Trans. Ind. Electron. 59, 2503 (2012).

[5] G. Pellegrino, A. Vagati, P. Guglielmi, and B. Boazzo, IEEE Trans. Ind. Electron. 59, 803 (2012).

[6] Q. Chen, G. Liu, W. Zhao, L. Sun, M. Shao, and Z. Liu, IEEE Trans. Ind. Electron. 61, 6615 (2014).

[7] W. Zhao, M. Cheng, K. T. Chau, J. Ji, and R. Cao, IEEE Trans. Ind. Electron. 60, 151 (2013).

[8] W. Zhao, M. Cheng, X. Zhu, W. Hua, and X. Kong, IEEE Trans. Ind. Electron. 55, 1739 (2008).

[9] W. Zhao, K. T. Chau, M. Cheng, J. Ji, and X. Zhu, IEEE Trans. Ind. Electron. 57, 2134 (2010).

[10] W. Zhao, M. Cheng, W. Hua, H. Jia, and R. Cao, IEEE Trans. Ind. Electron. 58, 1926 (2011).

[11] S. A. Hong, J. Y. Choi, and S. M. Jang, J. Magn. 19, 84 (2014).

[12] Z. Azar, Z. Q. Zhu, and G. Ombach, IEEE Trans. Magn. 48, 2650 (2012).

[13] S. H. Lee, Y. J. Kim, K. S. Lee, and S. J. Kim, J. Magn. 20, 444 (2015).

[14] J. H. Kim, J. M. Seo, H. K. Jung, and C. Y. Won, J. 
Magn. 19, 411 (2014).

[15] H. S. Chen, D. G. Dorrell, and M. C. Tsai, IEEE Trans. Magn. 46, 3664 (2010).

[16] K. Wang, Z. Q. Zhu, and G. Ombach, IEEE Trans. Magn.
50, 8100210 (2014).

[17] K. Wang, Z. Q. Zhu, G. Ombach, and W. Chlebosz, IEEE Trans. Ind. Electron. 61, 5047 (2014). 